\title{
ESTUDIO TEÓRICO DEL PLASMÓN EN NANOESFERAS DE ORO
}

\author{
Ana María Osorio Anaya ${ }^{* a}$, Joaquín J. Manrique Fajardo ${ }^{a}$, Óscar Cornejo Sánchez
}

\begin{abstract}
RESUMEN
En el presente trabajo se realizó la simulación del comportamiento plasmónico de nanoesferas de oro con diámetros de 10, 20, 30, 40, 50 y $60 \mathrm{~nm}$ en medio acuoso frente a la luz en el rango de longitudes de onda de 400 a $650 \mathrm{~nm}$. Se observó una mayor contribución de la dispersión al espectro de extinción en función del tamaño de nanoesferas, el cual presentó un comportamiento exponencial.
\end{abstract}

Palabras clave: nanoesferas de oro, plasmón, aproximación de dipolo discreto.

\section{THEORETICAL STUDY OF THE PLASMON IN GOLD NANOSPHERES}

\begin{abstract}
In the present work the simulation of the plasmonic behavior of gold nanospheres with diameters of 10, 20, 30, 40, 50 y $60 \mathrm{~nm}$ in aqueous medium against light with wavelenghts ranging from 400 to $650 \mathrm{~nm}$ was done. A greater contribution of the scattering to the extinction spectrum was observed as a function of the size of the nanosphere, which exhibited an exponential behavior.
\end{abstract}

Key words: gold nanospheres, plasmon, discret dipole aproximation.

\section{INTRODUCCIÓN}

En 1951, Turkevich y col. ${ }^{1}$ realizaron el estudio de los procesos de nucleación y desarrollo del oro coloidal, encontrando diversos tamaños y formas del metal, en función a la temperatura, concentración del reductor, entre otros factores, hoy en día este excelente estudio sirvió y sirve de base a la química computacional para realizar estudios teóricos de las propiedades ópticas que presentan las nanopartículas de oro al interaccionar con la luz, las cuales, en nivel experimental, presentan una variedad de colores debido al tamaño y forma.

\footnotetext{
a Laboratorio de Nanotecnología e innovación tecnologíca, Facultad de Química e Ingeniería Química, Universidad Nacional Mayor de San Marcos, Calle Germán Amézaga 375, Lima, Perú.

*aosorio@unmsm.edu.pe
} 
Nanopartículas de metales nobles como oro, platino y plata exhiben comportamiento de alta absorción y dispersión de luz, este es dependiente de su tamaño, forma y composición. Las nanopartículas de oro y plata muestran respuestas espectrales únicas debido a que en longitudes de ondas específicas llevan a los electrones libres o gas de electrones de las nanopartículas metálicas a oscilar colectivamente. De este comportamiento se genera la resonancia de plasmón de superficie localizada (LSPR), la cual está confinada a un volumen pequeño en la vecindad cercana de la nanopartícula aislada ${ }^{2}$.

Desde el punto de vista teórico, los fenómenos de interacción entre la radiación electromagnética y las nanopartículas metálicas generan un movimiento colectivo de los electrones de las nanopartículas dentro de un medio dieléctrico a una cierta frecuencia de resonancia, la LSPR genera dos efectos, la pérdida de calor debido a interacciones con la red de los átomos metálicos, originando la absorción de la luz y el desplazamiento de los electrones libres de carga negativa respecto al núcleo pesado del metal de carga positiva, generando un dipolo oscilante que emite radiación electromagnética a la misma frecuencia de la oscilación, según se observa en la figura 1, de tal forma que se genera fenómenos de dispersión de luz o scattering ${ }^{2,3}$.

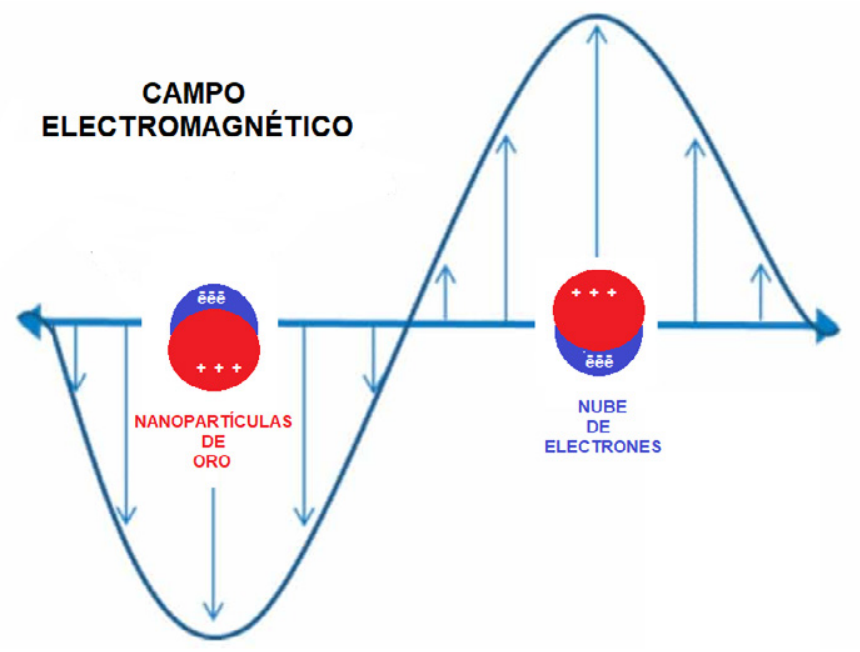

Figura 1. Resonancia de plasmón de superficie localizada (LSPR). Adaptado de Willets (2007).

Debido a sus propiedades ópticas las nanopartículas de oro presentan aplicaciones diversas. En el caso de la dispersión de luz es empleada en mapeo de imágenes y seguimiento de procesos biológicos dinámicos en células vivas y el fenómeno de absorción empleado como terapia fototérmica ${ }^{4,5}$. 
La aproximación de dipolo discreto (DDA) permite modelar las propiedades ópticas como dispersión y absorción de ondas electromagnéticas por partículas de geometría arbitraria y distintos índices de refracción, tanto como partículas aisladas como arreglos periódicos 1-d y 2-d mediante la representación de la partícula como un conjunto de puntos polarizables ${ }^{6}$.

La dispersión del campo magnético es resuelta para un conjunto de dipolos $(j=1, \ldots, N)$ con polarizabilidades $\alpha_{j}$, localizados en posisiones $r_{j}$. Cada dipolo tiene una polarizaciòn $P_{j}=\alpha_{j} E_{j}$ donde $E_{j}$ es el campo eléctrico en $r_{j}$ es debido a la onda incidente $E_{i n c, j}=E_{0} \exp \left(i k \cdot r_{j}-i \omega t\right)$ más la contribución de cada otro $N-1$ dipolos:

$$
E_{j}=E_{i n c, j}-\sum_{j k} A_{j k} P_{k}
$$

donde $-A_{j k} P_{k}$ es el campo eléctrico en $r_{j}$ debido al dipole $P_{k}$ en la posición $r_{k}$, incluyendo los efectos de retardación ${ }^{7}$.

La DDA se está aplicando para modelar no sólo las interacciones de la luz con nanopartículas esféricas de diversos tamaños, las que resultan con comportamientos isotrópicos frente a la luz, sino también a medida que se van conociendo nuevas vías de síntesis de nanopartículas metálicas para diversas aplicaciones, se proponen cálculos teóricos como para nanoelipsoides, nanocilindros y nanobarras, cuyas propiedades ópticas son anisotrópicas, para nanopartículas esféricas se muestra una sola banda de absorción y para partículas tipo nanorod se presentan dos bandas de absorción ${ }^{8,9}$.

Como se sabe, las nanopartículas al interaccionar con la luz tienen la probabilidad de que un fotón incidente sea absorbido o dispersado, tal como se aprecia en la figura 2, este fenómeno óptico puede expresarse en forma cuantitativa a través de los valores de la sección eficaz de absorción ( $\sigma a b s)$ y de dispersión o scaterring ( $\sigma s c a)$. Al aumentar la sección eficaz de absorción o de dispersión, aumenta la probabilidad de que sucedan estos fenómenos. La suma de los efectos de absorción y dispersión se denomina extinción $(\boldsymbol{\sigma e x t}=\sigma a b s+\sigma s c a)^{3}$.

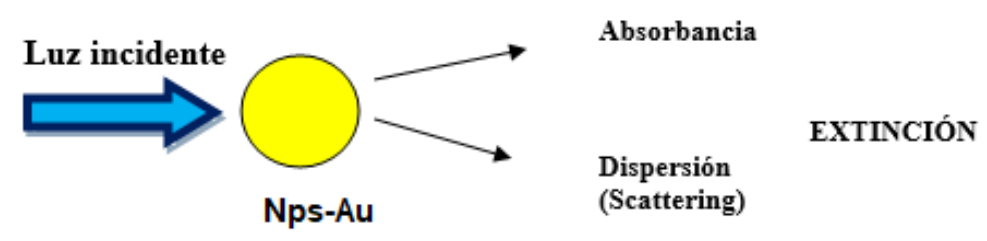

Figura 2. Extinción en función de la absorbancia y la dispersión. 


\section{DETALLES DE LA SIMULACIÓN COMPUTACIONAL}

La respuesta óptica de nanoesferas de oro de 10, 20, 30, 40, 50 y $60 \mathrm{~nm}$ de diámetro fue calculada bajo el método de aproximación de dipolo discreto (DDA) empleando el código por Draine y Flatau $^{10}$ y haciendo uso de los algoritmos: gradiente conjugada con estabilización implementado en la subrutina ZBCG2 por Botchev ${ }^{11}$ y GPFA FFT por Temperton ${ }^{12}$.

Se emplearon las constantes ópticas reportadas por Johnson y Christy ${ }^{12}$, el medio rodeando a la nanoesfera fue representado por agua con un índice de refracción de 1,33. El desarrollo computacional se llevó a cabo con luz incidente simulada en el rango de 400 a $650 \mathrm{~nm}$.

Las simulaciones se realizaron en una computadora core i7 7700K a $4.30 \mathrm{GHz}$ con $16 \mathrm{~GB}$ de RAM. Los cálculos tardaron alrededor de 10 horas.

Se graficaron los datos de simulación para cada tamaño de nanoesfera de oro y se registran los datos en la tabla $\mathrm{N}^{\circ} 1$ y tanto ésta como los gráficos 9 y 10 se emplearán para la discusión.

\section{RESULTADOS Y DISCUSIÓN}

Los espectros de extinción $\left(\mathrm{Q}_{\mathrm{ext}}\right)$, absorción $\left(\mathrm{Q}_{\mathrm{abs}}\right)$ y dispersión $\left(\mathrm{Q}_{\mathrm{sca}}\right)$ para nanoesferas de oro de distintos tamaños: 10, 20, 30, 40, 50 y $60 \mathrm{~nm}$ en medio acuoso, como se muestran en las figuras 3-8 observándose la presencia de una única banda de plasmón.

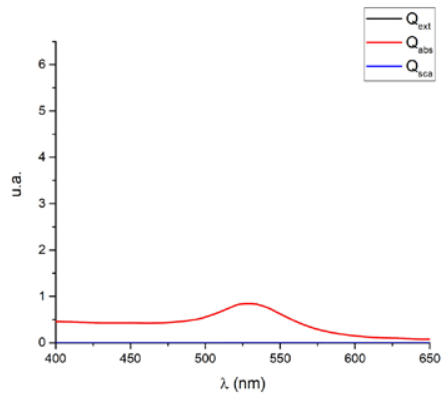

Figura 3. Espectro de extinción, absorción y dispersión nanoesfera de oro de 10nm.

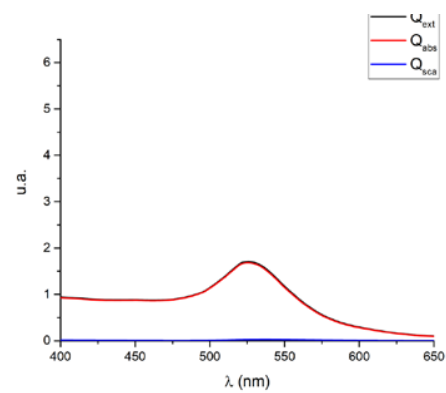

Figura 4. Espectro de extinción, absorción y dispersión nanoesfera de oro de $20 \mathrm{~nm}$. 


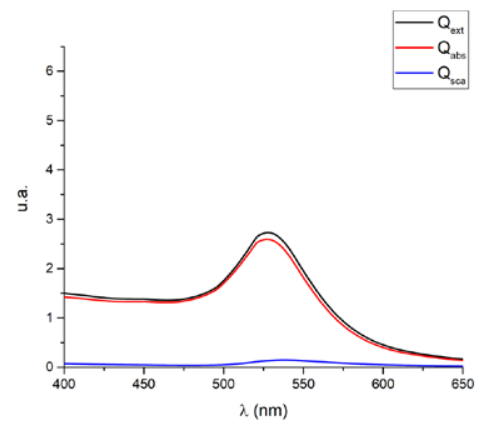

Figura 5. Espectro de extinción, absorción y dispersión nanoesfera de oro de 30nm.

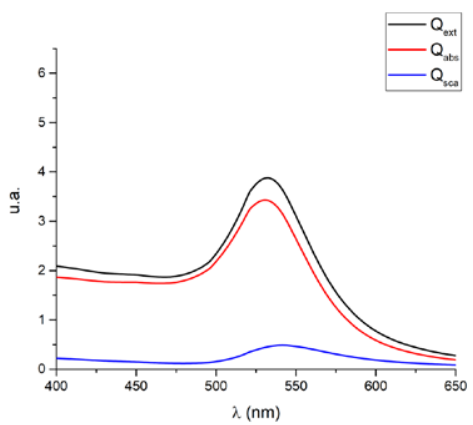

Figura 6. Espectro de extinción, absorción y dispersión nanoesfera de oro de 40nm.

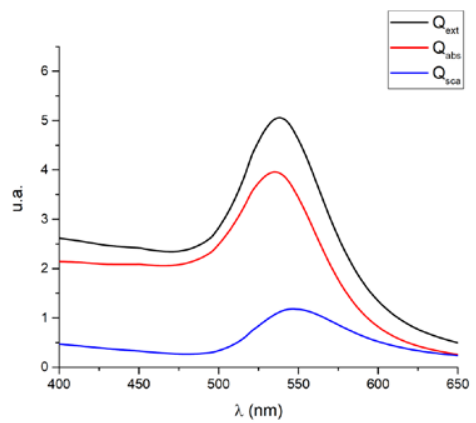

Figura 7. Espectro de extinción, absorción y dispersión nanoesfera de oro de 50nm. 


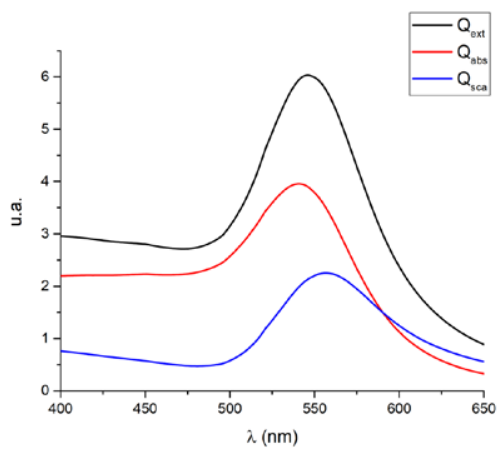

Figura 8. Espectro de extinción, absorción y dispersión nanoesfera de oro de $60 \mathrm{~nm}$.

De los espectros recolectados se observó un corrimiento rojo del $\lambda_{\max }$ de las nanoesferas a medida del aumento de diámetro de esta concordando con lo reportado en la literatura ${ }^{13,14}$, este comportamiento se origina por una serie de oscilaciones multipolo para la sección transversal de las nanoesferas.

Los máximos de las bandas de plasmón de las nanoesferas en sus respectivas longitudes de ondas se encuentran resumidos en la tabla 1. Jain et al. ${ }^{15}$ han reportado cálculos para nanoesferas de oro de 20, 40 y 80 , obteniéndose valores similares a los reportados en el presente trabajo.

Tabla 1. Relación de longitudes de onda y máximos de banda en función al tamaño de las nanopartículas de oro.

\begin{tabular}{ccc}
\hline $\begin{array}{c}\text { Diámetro } \\
(\mathrm{nm})\end{array}$ & $\begin{array}{c}\text { Longitud de onda } \\
(\mathrm{nm})\end{array}$ & $\begin{array}{c}\text { Máximo de banda } \\
(\text { u.a. })\end{array}$ \\
\hline 10 & 528 & 0,84855 \\
20 & 525 & 1,70890 \\
30 & 527 & 2,73010 \\
40 & 532 & 3,88000 \\
50 & 538 & 5,05900 \\
60 & 546 & 6,03410 \\
\hline
\end{tabular}

Según la tabla 1, tanto las longitudes de onda como el máximo de banda de absorción se incrementan con el tamaño de las nanopartículas esféricas, ello se ha observado en trabajos experimentales realizados en nuestro laboratorio y en otros trabajos publicados, estos estudios son de mucho interés para evaluar el comportamiento del tamaño y la forma para aplicaciones biomédicas en partículas para su estudio en tratamiento de células cancerígenas ${ }^{4,15}$. 


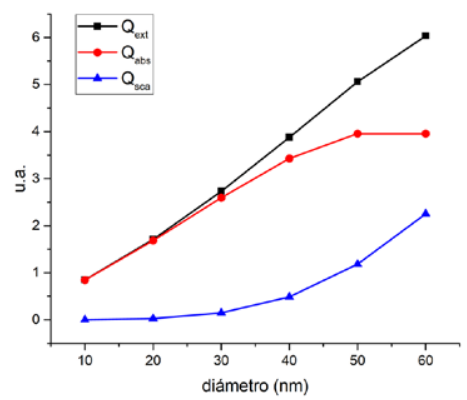

Figura 9. Contribución de la absorbancia y dispersión (scattering).

Se observó un incremento en la contribución de la dispersión en el espectro de extinción a medida que se incrementa el diámetro de la nanoesfera de oro concordando con lo reportado por Jain ${ }^{14}$, presentando un comportamiento exponencial la contribución del espectro de dispersión tal como se observa en la figura 10.
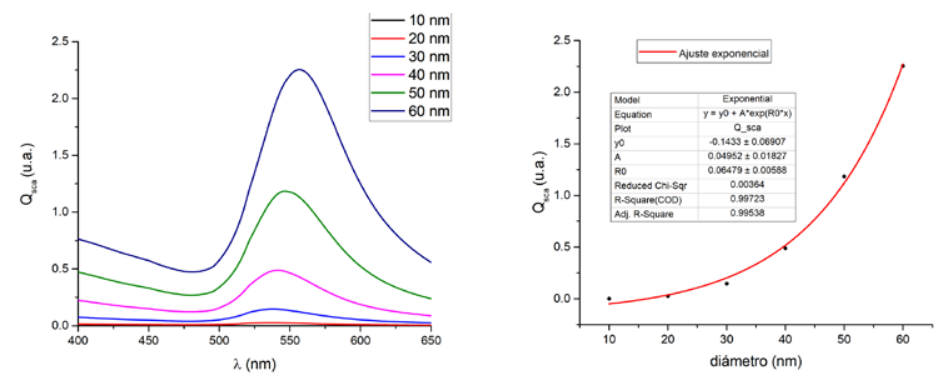

Figura 10. Contribución y ajuste de la dispersión (scattering).

\section{CONCLUSIONES}

Según los resultados de simulación obtenidos en el presente trabajo se puede concluir que la oscilación dipolar del desplazamiento de los electrones respecto a su núcleo metálico positivo resuena con la luz incidente y la respuesta del comportamiento de oscilación colectiva depende del tamaño y la forma de las nanopartículas, en este caso el estudio se realizó sobre partículas esféricas de oro de $10 \mathrm{~nm}$ hasta $60 \mathrm{~nm}$, cuya longitud de onda de absorción se incrementa a medida que aumenta el tamaño de las nanopartículas desde $528 \mathrm{~nm}$ hasta $546 \mathrm{~nm}$. 


\section{AGRADECIMIENTO}

Agradecemos al Vicerrectorado de Investigación por el financiamiento del Proyecto de Investigación No C17071311 de la Facultad de Química e Ingeniería Química de la Universidad Nacional Mayor de San Marcos

\section{REFERENCIAS BIBLIOGRÁFICAS}

1. Turkevich J, Stevenson PC, Hillier J. A study of the nucleation and growth processes in the synthesis of colloidal gold. Discuss Faraday Soc. 1951; 11:55-75.

2. Willets KA, Van Duyne RP. Localized Surface Plasmon Resonance Spectroscopy and Sensing. Annu Rev Phys Chem. 2007;58(1):267-297.

3. Garate O, Veiga L, Lloret P, Ybarra G. Introducción a los fenómenos ópticos de nanopartículas metálicas a través simulaciones computacionales en línea. Educ Química. 2019; 30(1):31-41.

4. Jain PK, Huang X, El-Sayed IH, El-Sayed MA. Noble Metals on the Nanoscale: Optical and Photothermal Properties and Some Applications in Imaging, Sensing, Biology, and Medicine. Acc Chem Res. 2008;41(12):1578-1586.

5. Link S, El-Sayed MA. Optical Properties and Ultrafast Dynamics of Metallic Nanocrystals. Annu Rev Phys Chem. 2003; 54(1):331-366.

6. Draine BT, Flatau PJ. Discrete-Dipole Approximation For Scattering Calculations. J Opt Soc Am A. 1994; 11(4):1491-1499.

7. Draine BT, Flatau PJ. Discrete-dipole approximation for periodic targets: theory and tests. J Opt Soc Am A. 2008;25(11):2693-2703.

8. Eustis S, El-Sayed MA. Why gold nanoparticles are more precious than pretty gold: Noble metal surface plasmon resonance and its enhancement of the radiative and nonradiative properties of nanocrystals of different shapes. Chem Soc Rev. 2006;35(3):209-217.

9. Tuersun P, Yusufu T, Yimiti A, Sidike A. Refractive index sensitivity analysis of gold nanoparticles. Optik (Stuttg). 2017; 149:384-390.

10. Draine BT, Flatau PJ. User Guide for the Discrete Dipole Approximation Code DDSCAT 7.3. [Internet]. 2013. [Consultado 12 junio 2019]. Disponible en: http://arxiv.org/ abs/1305.6497

11. Botchev MA. Subroutine ZBCG2. [Internet]. 2001. [Consultado 15 julio 2019]. Disponible en: http://www.staff.science.uu.nl/ vorst102/zbcg2.f90.

12. Temperton C. A Generalized Prime Factor FFT Algorithm for any $N=2 p 3 q$ 5r. SIAM J Sci Stat Comput. 1992;13(3):676-686.

13. Link S, El-Sayed MA. Size and Temperature Dependence of the Plasmon Absorption of Colloidal Gold Nanoparticles. J Phys Chem B. 1999; 103(21):4212-4217.

14. Link S, El-Sayed MA. Shape and size dependence of radiative, non-radiative and photothermal properties of gold nanocrystals. Int Rev Phys Chem. 2000;19(3):409-453.

15. Jain PK, Lee KS, El-Sayed IH, El-Sayed MA. Calculated absorption and scattering properties of gold nanoparticles of different size, shape, and composition: Applications in biological imaging and biomedicine. J Phys Chem B. 2006;110(14):7238-7248. 